\title{
Combination Therapy with Clindamycin and Rifampicin for Hidradenitis Suppurativa: A Series of 116 Consecutive Patients
}
G. Gener ${ }^{a}$
F. Canoui-Poitrine ${ }^{b, d, e}$
J.E. Revuz ${ }^{a}, d$
O. Faye ${ }^{f}$
F. Polia
G. Gabison ${ }^{a}$
F. Pouget ${ }^{a}$
C. Viallette ${ }^{c}$
P. Wolkenstein ${ }^{a, d}$
S. Bastuji-Garin b, d

Services de a Dermatologie et ${ }^{b}$ Santé Publique et ${ }^{c}$ Unité de Recherche Clinique, Groupe Henri-Mondor Albert-Chenevier, AP-HP, dUniversité Paris 12, Faculté de Médecine, LIC EA 4393 Créteil, et ${ }^{\text {e}}$ Hospices Civils de Lyon,

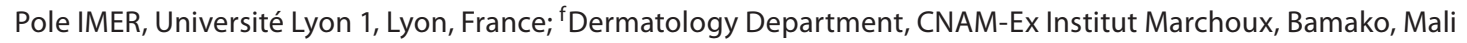

\section{Key Words}

Hidradenitis suppurativa $\cdot$ Rifampicin · Clindamycin •

Sartorius score $\cdot$ Quality of life

\begin{abstract}
Background: Antibiotics are frequently used to treat hidradenitis suppurativa (HS); however, few data on their efficacy are available. Objective: To evaluate the efficacy of a combination of systemic clindamycin (300 mg twice daily) and rifampicin ( $600 \mathrm{mg}$ daily) in the treatment of patients with severe HS. Methods: Patients $(\mathrm{n}=116)$ who received this combination were studied retrospectively. The main outcome measure was the severity of the disease, assessed by the Sartorius score, before and after 10 weeks of treatment. Results: The Sartorius score dramatically improved at the end of treatment (median $=29$, interquartile range $=14.5$, vs . median $=14.5$, interquartile range $=11 ; p<0.001$ ), as did other parameters of severity as well as the quality of life score. Eight patients (6.9\%) stopped the treatment because of side effects. Conclusion: The combination of clindamycin and rifampicin is effective in the treatment of severe HS.
\end{abstract}

Copyright $\odot 2009$ S. Karger AG, Basel
(C) 2009 S. Karger AG, Basel

$1018-8665 / 09 / 2192-0148 \$ 26.00 / 0$

Fax +4161306 1234

E-Mail karger@karger.ch

www.karger.com
Accessible online at: www.karger.com/drm

\section{Introduction}

Hidradenitis suppurativa (HS) is a chronic inflammatory disease, characterized by painful nodules and draining sinus tracts, affecting apocrine areas of the skin. The prevalence of HS has been estimated to be $1 \%$ of the general population $[1,2]$. Management of HS is difficult; relapses and chronicity contribute to produce a severe impact upon the quality of life [3]. Numerous treatments, such as topical and systemic antibiotics, oral zinc, hormonal therapies, oral retinoids, immunosuppressant agents, anti-tumour-necrosis-factor biologicals and surgical interventions have been used, but their efficacy has seldom been assessed.

HS is not primarily an infectious disease, although bacterial infection is often present. A large variety of micro-organisms has been isolated from the lesions; the most frequently isolated pathogens are Staphylococcus aureus, Gram-negative rods [4-6] and anaerobic bacteria $[5,7]$. Unfortunately the clinical significance of superficial and even deep bacterial sampling is very low in the clinical setting [8]. Representative flora has been identified using carbon dioxide laser evaporation with bacterial sampling at each level but this is not convenient in common practice [9]. Accordingly the choice of an antibiotic combination cannot be based on bacterial sampling. 
Table 1. Sartorius score modified by J.E. Revuz

\begin{tabular}{|c|c|c|c|c|}
\hline \multicolumn{2}{|r|}{ Anatomical region involved: armpit, breast, inguinofemoral, perianal and perineal areas } & - & $\times 3$ & \\
\hline \multirow{3}{*}{2} & - abscess or fistulas & & $\begin{array}{r}\times 2 \\
\times 4\end{array}$ & \\
\hline & - hypertrophic scars & - & $\times 1$ & \\
\hline & - others (folliculitis, pustules, ...) & _- & $\times 0.5$ & \\
\hline \multirow[t]{2}{*}{4} & Are all lesions clearly separated by normal skin? $($ yes $=0 ;$ no $=6)$ & | & $\times 1$ & \\
\hline &  & & & \\
\hline
\end{tabular}

Short clinical series have claimed the efficacy of the combination of systemic clindamycin and rifampicin in folliculitis decalvans [10] and, subsequently, in 10 patients with HS [11]. In our centre, this combination is widely used for severe HS, using a treatment cycle of 10 weeks. The aim of our study was to evaluate retrospectively the efficacy of this combination of antibiotics in our cohort of patients with HS.

\section{Methods}

\section{Patients}

Consecutive patients with HS were examined in our centre by the same investigator (J.R.), from September 2003 to September 2007 ( $n=373)$. Diagnostic criteria were: (1) presence of typical lesions, i.e. deep-seated nodules (blind boils), abscesses and/or fibrosis, (2) location in typical areas, i.e. armpit and groin (and, secondarily, breast, buttocks and perineum), and (3) evolution, with relapses and chronicity. The above 3 criteria were considered necessary to establish the diagnosis.

At the first visit (week 0, W0), a standardized form was used to prospectively record demographic characteristics, socioprofessional data, smoking habits, history of the disease and a family history of HS. The body mass index (BMI) was computed. Active acne at the time of examination and a personal history of severe acne were both recorded separately. The anatomical zones which had been involved at least once in the history of the patient were recorded: 'typical locations', i.e. axillae, breast, genitofemoral area, buttocks, perianal and perineal areas; 'atypical locations', i.e. ears, chest, and associated follicular diseases, such as dissecting folliculitis of the scalp and pilonidal cysts. The type of lesions, e.g. nodules, abscesses/sinus/fistulas, hypertrophic scars or folliculitis, was described.

Patients treated with the combination of rifampicin and clindamycin were extracted from our database. The decision to use this treatment was taken by one of us (J.R.), based on the degree of severity and inflammation present. The regimen was clindamycin $300 \mathrm{mg}$ twice daily and rifampicin $600 \mathrm{mg}$ once dai- ly in the morning during a period of 10 weeks. Women using contraceptive pills were systematically informed at the first visit that they had to use mechanical contraception.

\section{Data Collection}

A standardized form was used to collect data before the antibiotic treatment was initiated (W0) and at the end of the 10-week treatment (W10). Disease activity was systematically assessed by the Sartorius severity score $[12,13]$ (table 1). Hurley's classification [14] (table 2) was assessed for each zone involved; the final grade was the grade of the most severely affected zone. Maximal pain score and maximal suppuration score were assessed by the patient for the period of the preceding month, with a numerical scale from 0 to 10 . The number of painful days per month and the number of days with suppuration were evaluated by the patient, with the result being recorded in 4 categories: none, $<15$ days, $\geq 15$ days or permanent. The patient's quality of life was assessed using a skin-disease-specific tool, the Skindex-France questionnaire [3]. However, as this tool was only introduced belatedly, this assessment involved only the patients included later in the database.

The treatment was also assessed by the patients: at W10, they were asked for their perception of the result of the treatment on HS symptoms (i.e. very good, good, stable, worse). Finally, they were asked about potential side effects during the treatment, i.e. diarrhoea, abdominal pain or others.

\section{Statistical Analysis}

Initial characteristics and disease activity of the 116 patients who used the combination therapy were described and compared to those of patients who had been treated with another therapy. Patients whose W10 evaluation was missing $(\mathrm{n}=46)$ were also compared with the 70 patients whose W10 data were available. Continuous data are presented as means ( \pm 1 standard deviation) or medians (with interquartile range, IQR $=\mathrm{Q} 3-\mathrm{Q} 1$ ) for non-normally distributed variables and were compared by using the Student $t$ test or the non-parametric Wilcoxon-Mann-Whitney $U$ test, as appropriate. Categorical data are presented as numbers (with percentages) and compared by using the $\chi^{2}$ or Fisher exact test, as appropriate. 
Fig. 1. Flow chart.

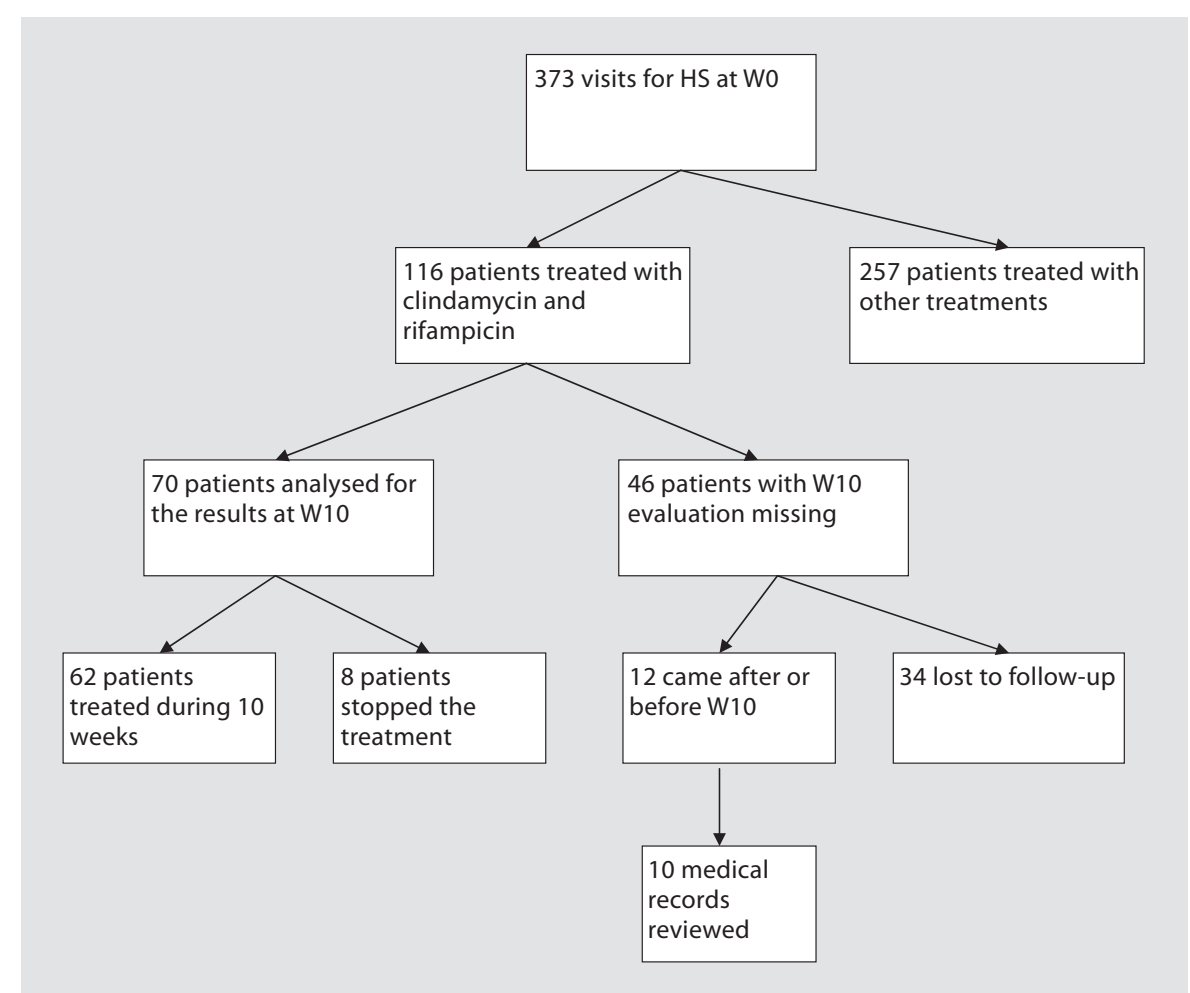

The main measure of outcome was the severity of the disease as assessed by the Sartorius score; the secondary outcome measures were Hurley's classification, intensity and duration of pain and suppuration, and quality of life scores. The measures before and after treatment were compared using the Wilcoxon matchedpair signed-rank test or test of marginal homogeneity (StuartMaxwell) for paired data, as appropriate. As data of 46 patients were not available at W10, we also analysed the main outcome measure using the 'worst-case hypothesis', i.e. assuming that these 46 patients had no improvement of the Sartorius score.

All comparisons were two-sided, and a $\mathrm{p}$ value $<0.05$ indicated a statistically significant difference. Data were analysed using the Stata Statistical software (Stata Corp. 2003, release 8.2; College Station, Tex., USA).

\section{Results}

\section{Patient Characteristics}

Among the 373 patients registered in the database, 116 (31\%) received the combination therapy. W10 assessment was missing for 46 patients: 34 lost to follow-up and 12 whose evaluation was performed before or after W10. Therefore, data before and after treatment were available for $70(60 \%)$ patients (fig. 1).

Table 3 describes the initial clinical characteristics of patients treated by combination therapy of clindamycin-
Table 2. Hurley's classification [14]

Grade I Abscess formation, single or multiple, without sinus tracts and cicatrization

Grade II Recurrent abscesses with tract formation and cicatrization; single or multiple, widely separated lesions

Grade III Diffuse or near-diffuse involvement, or multiple interconnected tracts and abscesses across entire area

rifampicin. Thirty-one men and 85 women were included (sex ratio 0.4 ); their mean age was 33 years $( \pm 10)$. The mean BMI was $27( \pm 7)$. The percentage of active cigarette smokers was 79 . The mean self-reported disease duration was 11 years $( \pm 9)$. The median score of the numerical scale for pain and suppuration was $7(\mathrm{IQR}=3)$. The median Sartorius score was $28(\mathrm{IQR}=14.5)$. There was no significant difference in the baseline characteristics, including the clinical severity as assessed by the Sartorius score, Hurley's classification, and intensity and duration of pain and suppuration recorded at the first visit, between the 70 patients who were followed up and the 46 who were not (data not shown). 
Table 3. Comparison of initial characteristics of patients treated with rifampicin and clindamycin and those treated by other treatments

\begin{tabular}{|c|c|c|c|}
\hline & $\begin{array}{l}\text { Combination } \\
\text { rifampicin and } \\
\text { clindamycin } \\
(\mathrm{n}=116)\end{array}$ & $\begin{array}{l}\text { Other } \\
\text { treatments } \\
(\mathrm{n}=257)\end{array}$ & $\mathrm{p}$ \\
\hline Age at examination, years & $33 \pm 10$ & $32 \pm 9$ & 0.88 \\
\hline Men & $31(27)$ & $57(22)$ & 0.34 \\
\hline Weight, kg & $77 \pm 18$ & $71 \pm 17$ & $<0.001$ \\
\hline BMI & $27 \pm 6$ & $25 \pm 6$ & 0.01 \\
\hline Current smokers & $92(79)$ & $192(75)$ & 0.30 \\
\hline Cigarettes per day & $15(8)$ & $15(8)$ & 0.92 \\
\hline Active acne & $14(12)$ & $31(12)$ & 1.00 \\
\hline History of acne & $29(25)$ & $67(26)$ & 0.83 \\
\hline Length of disease, years & $11 \pm 9$ & $11 \pm 8$ & 0.40 \\
\hline Sartorius score median & $28[14.5]$ & $14[10]$ & $<0.0001$ \\
\hline \multicolumn{4}{|l|}{ Hurley's classification } \\
\hline I & $51(44.0)$ & $187(81.6)$ & \multirow{3}{*}{$<0.001$} \\
\hline II & $57(49.1)$ & $37(16.2)$ & \\
\hline III & $8(6.9)$ & $5(2.2)$ & \\
\hline Max. pain/10 med. & $7[3]$ & $4[6]$ & 0.0001 \\
\hline Max. suppuration/10 med. & 1. $7[3]$ & $3[6]$ & 0.0001 \\
\hline \multicolumn{4}{|c|}{ Number of days per month with pain } \\
\hline None & $3(2.6)$ & $7(3.1)$ & \multirow{4}{*}{$<0.001$} \\
\hline$<15$ & $35(30.4)$ & $154(68.8)$ & \\
\hline$\geq 15$ & $27(23.5)$ & $37(16.5)$ & \\
\hline Permanent & $50(43.5)$ & $26(11.6)$ & \\
\hline \multicolumn{4}{|c|}{ Number of days per month with suppuration } \\
\hline None & $5(4.4)$ & $81(31.9)$ & \multirow{4}{*}{$<0.001$} \\
\hline$<15$ & $30(26.0)$ & $115(45.1)$ & \\
\hline$\geq 15$ & $23(20.0)$ & $23(9.0)$ & \\
\hline Permanent & $57(49.6)$ & $36(14.0)$ & \\
\hline
\end{tabular}

Continuous data are expressed as means \pm SD except when otherwise indicated; categorical data are expressed as numbers, with percentages in parentheses; figures in square brackets indicate IQR. $p$ values were determined by the $\chi^{2}$ or Fisher exact test for categorical variables, by the Student $t$ test for normally distributed continuous variables (BMI) and by the Wilcoxon-MannWhitney $U$ test for non-normally distributed continuous variables (age, Sartorius score).

The 257 patients who did not receive the combination therapy had similar demographic characteristics, but had a lower BMI and were less severely affected than the 116 who received the combination of antibiotics (table 3).

\section{Therapeutic Results}

When considering the 70 patients whose $\mathrm{W} 0$ and $\mathrm{W} 10$ data were available (table 4), the main outcome measure (the severity of the disease assessed by the Sartorius score) dramatically and significantly decreased at the end of the treatment $($ median $=29, \mathrm{IQR}=14.5$, vs. median $=14.5$, $\mathrm{IQR}=11 ; \mathrm{p}<0.001)$. A significant decrease in the Sartorius score (median $=28, \mathrm{IQR}=14.5$, at $\mathrm{W} 0$ and median $=$ $19.3, \mathrm{IQR}=15.5$, at $\mathrm{W} 10 ; \mathrm{p}<0.001)$ was also observed when assuming that the 46 patients whose W10 data were not available had no improvement of the Sartorius score (i.e. 'worst-case hypothesis').

Eight patients (11\%) had complete remission (Sartorius score $=0)$. One patient demonstrated no improvement, and the severity of HS increased in 2 patients.

Similarly, a higher proportion of patients were classified before treatment into the severest Hurley grades, i.e. II and III, as compared to after treatment $(53.5 \%$ at $\mathrm{W} 0$ and $34.5 \%$ at $\mathrm{W} 10 ; \mathrm{p}=0.018$ ). The median of the maximum pain score was $7(\mathrm{IQR}=3)$ before treatment versus $3(\mathrm{IQR}=5)$ after treatment $(\mathrm{p}<0.001)$, and the median of the maximum suppuration score was $6(\mathrm{IQR}=4)$ versus $2(\mathrm{IQR}=6 ; \mathrm{p}<0.001)$.

Among the 70 patients, $46(66 \%)$ considered the result of treatment as very good, $19(27 \%)$ as good, $4(6 \%)$ as stable, and 1 patient complained of a worsening of the disease.

Twenty-nine patients filled in the skin-disease-specific quality of life questionnaire (Skindex-France). The score of each dimension (emotion, symptoms and function) was significantly improved after treatment (table 4).

\section{Adverse Events}

Ten out of the 70 patients (14\%) complained of side effects, mostly nausea, diarrhoea and abdominal pain. Eight had to stop the antibiotic treatment (11.4\%), 6 of whom because of digestive symptoms; all recovered without any specific treatment after stopping the antibiotic combination. One patient stopped because of a skin eruption 21 days after the beginning of the treatment and 1 because of absence of improvement after 7 weeks of treatment. Six of these 8 patients recorded the results of the antibiotic combination treatment as good or very good.

Among the 46 patients whose W10 data were not available, 10 came more than 10 days before or after the planned visit at W10. Their medical records were reviewed. Seven patients felt that they had improved, i.e. 4 with very good and 3 with good results.

\section{Discussion}

After 10 weeks of treatment with the antibiotic combination of clindamycin and rifampicin in severely affected patients with HS, we observed a dramatic improve- 
Table 4. Changes in the Sartorius score, Hurley's classification, intensity and duration of pain and suppuration, and skin-disease-specific quality of life score (Skindex-France) before and after treatment with clindamycin and rifampicin

\begin{tabular}{|c|c|c|c|c|}
\hline & & W0 & W10 & $\mathrm{p}$ \\
\hline Sartorius score median & $(\mathrm{n}=70)$ & $29[14.5]$ & $14.5[11]$ & $<0.001$ \\
\hline \multicolumn{5}{|l|}{ Hurley's classification } \\
\hline $\mathrm{I}$ & & $27(46.6)$ & $38(65.5)$ & \multirow{3}{*}{0.018} \\
\hline II & & $27(46.6)$ & $18(31.0)$ & \\
\hline III & & $4(6.9)$ & $2(3.5)$ & \\
\hline Maximal pain/10 median & $(\mathrm{n}=69)$ & $7[3]$ & $3[5]$ & $<0.001$ \\
\hline Maximal suppuration/10 median & $(\mathrm{n}=70)$ & $6[4]$ & $2[6]$ & $<0.001$ \\
\hline \multicolumn{5}{|l|}{ Number of days per month with pain } \\
\hline None & & $2(3.4)$ & $6(10.2)$ & \multirow{4}{*}{$<0.001$} \\
\hline$<15$ & & $17(28.8)$ & $43(72.9)$ & \\
\hline$\geq 15$ & & $13(22.0)$ & $3(5.0)$ & \\
\hline Permanent & & $27(45.8)$ & $7(11.9)$ & \\
\hline \multicolumn{5}{|c|}{ Number of days per month with suppuration $(n=59)$} \\
\hline None & & $1(1.7)$ & $17(28.8)$ & \multirow{4}{*}{$<0.001$} \\
\hline$<15$ & & $10(17.0)$ & $22(37.3)$ & \\
\hline$\geq 15$ & & $13(22.0)$ & $5(8.5)$ & \\
\hline Permanent & & $35(59.3)$ & $15(25.4)$ & \\
\hline \multicolumn{5}{|l|}{ Skindex-France factors } \\
\hline Emotion/100 & $(\mathrm{n}=29)$ & $71 \pm 7$ & $49 \pm 9$ & $<0.001$ \\
\hline Symptoms/100 & $(\mathrm{n}=29)$ & $58 \pm 14$ & $34 \pm 8$ & $<0.001$ \\
\hline Function/100 & $(\mathrm{n}=29)$ & $57 \pm 10$ & $33 \pm 11$ & $<0.001$ \\
\hline
\end{tabular}

Continuous data are expressed as means \pm SD except when otherwise indicated; categorical data are expressed as numbers, with percentages in parentheses; figures in square brackets indicate IQR. $\mathrm{p}$ values were determined by the Wilcoxon matched-pair signed-rank test or test of marginal homogeneity (Stuart-Maxwell) for paired data.

ment of the disease activity as assessed by the Sartorius score, but also by other measurements (Hurley's classification, frequency and intensity of pain and suppuration). Furthermore, all dimensions of quality of life improved significantly. The results of a study on the same topic published in this issue of Dermatology are in agreement with ours: out of $34 \mathrm{HS}$ patients treated by the combination of clindamycin and rifampicin, the majority (82\%) had an improvement of their clinical features [15]. Conversely the proportion of side effects was substantially higher in the other study than in ours (38.2 vs. $14 \%$ ).

Our study has several limitations. It is an observational study and not a clinical trial. The decision to treat these 116 patients with the antibiotic combination had been taken by the principal investigator on clinical grounds, without any prior definition of 'inclusion criteria'. Analysis of their data, however, shows that as a group they were significantly more severely affected than the 257 who received another treatment. A limited number of before and after quality of life scores were filled out.
Moreover, the clinical endpoints of $40 \%(\mathrm{n}=46)$ of patients out of 116 could not be analysed after the treatment, leading to the possibility of a selection bias:

- 34 did not return at the end of treatment, because they were followed up by their referring physician and no data on the results were available; however, the initial characteristics of these 34 patients did not differ from the 70 who were analysed;

- 12 patients came for review before or after the planned 10 weeks, and they were not included in the analysis, even though the results of treatment seem to have been the same as for the 70 patients analysed.

No data about long-term follow-up and recurrences are given. In fact as this treatment was aimed at being suspensive only, a maintenance treatment with tetracyclines or zinc gluconate was prescribed at the end of the 10 -week regimen. The results of such maintenance treatment are not available at this time.

The Sartorius score, which we used to assess the efficacy of treatment, is not formally validated. However, our 
study shows a parallel between the evolution of the Sartorius score, the degree of intensity and duration of pain and suppuration, and the quality of life scores. Therefore, it seems relevant to use the Sartorius score to measure both the severity and the evolution of HS. Furthermore, the Sartorius score has been shown to correlate closely with the intensity and duration of pain and suppuration and with the Hurley classification in a series of $302 \mathrm{HS}$ patients [16].

The strengths of the study are that a large number of consecutive patients were included, the diagnosis of HS was assessed using stringent clinical criteria, and all patients were examined by the same investigator. A standardized form was used for the prospective collection of data.

The disease has a psychological impact, especially upon quality of life and mental health $[3,17,18]$. The skin-disease-specific quality of life questionnaire was significantly improved after the treatment.

Rifampicin is a broad-spectrum antibacterial agent that inhibits the growth of the majority of Gram-positive bacteria as well as of many Gram-negative micro-organisms [19]. It is highly active against both $S$. aureus and coagulase-negative staphylococci. When the drug was used alone, rapid emergence of resistance limited its use, except in association with another antistaphylococcal drug [20]. Clindamycin is a lincosamide antibiotic active against Gram-positive cocci and most anaerobic bacteria [21]. One trial found topical clindamycin superior to its vehicle [22], while another showed no difference between topical clindamycin and systemic tetracycline [23]. Clindamycin helps to prevent bacterial resistance against rifampicin and covers a broad antibacterial spectrum. The combination of systemic clindamycin $(600 \mathrm{mg}$ daily) and rifampicin (600 mg daily) was given for 10 weeks, with success, to 14 patients with long-lasting HS [11]; 10 patients achieved remission. As in our study, diarrhoea was the most frequent side effect: minocycline was substituted for clindamycin in 2 patients, and the treatment was stopped in 4 cases. Both antibiotics were used at a relatively low dose in the same way as they had been used by Mendonça and Griffiths [11]. This way of using rifampicin and clindamycin is different from the way it is used in acute infectious diseases where higher daily doses are used during shorter periods: here it is a longterm treatment, i.e. 10 weeks for a disease which is not primarily infectious. Clindamycin and rifampicin have both antibacterial and anti-inflammatory effects [2428]. Our data cannot elucidate the exact mechanism of the beneficial effect of clindamycin and rifampicin; nonetheless, we can hypothesize that their efficacy in HS, which is an inflammatory disease with bacterial superinfection, could be due to both antibacterial and anti-inflammatory properties.

Recently, several studies concerning anti-tumour-necrosis-factor treatment with infliximab [29-34], etanercept $[35,36]$ or adalimumab $[37,38]$ have reported a dramatic response in HS. A prospective trial comparing the efficacy of the combination clindamycin-rifampicin to anti-tumour-necrosis-factor biologicals in HS would help to assess the respective role of these therapeutic approaches.

In conclusion, these results suggest that the antibiotic combination of clindamycin and rifampicin significantly improves the clinical features and the quality of life of patients with severe HS. Prospective randomized controlled trials are needed to confirm these results.

\section{References}

1 Jemec GBE, Heidenheim M, Nielsen NH: The prevalence of hidradenitis suppurativa and its potential precursor lesions. J Am Acad Dermatol 1996;35:191-194.

$\checkmark 2$ Revuz JE, Canoui-Poitrine F, Wolkenstein P, Viallette C, Gabison G, Pouget F, et al: Prevalence and factors associated with hidradenitis suppurativa: a case control study. J Am Acad Dermatol 2008;59:596-601.

3 Wolkenstein P, Loundou A, Barrau K, Auquier P, Revuz J: Quality of life impairment in hidradenitis suppurativa: a study of 61 cases. J Am Acad Dermatol 2007;56:621623.
4 Jemec GBE, Heidenheim M, Nielsen NH: Hidradenitis suppurativa. Cutan Med Surg 2003;7:47-56.

5 Brook I, Frazier EH: Aerobic and anaerobic microbiology of axillary hidradenitis suppurativa. J Med Microbiol 1999;48:103-105.

6 Jemec GBE, Faber M, Gutschik E, Wendelboe P: The bacteriology of hidradenitis suppurativa. Dermatology 1996;193:203-206.

7 Leach RD, Eykyn SJ, Phillips I, et al: Anaerobic axillary abscess. Br Med J 1979;2:5-7.

8 Oprica C, Nord CE: Bacteriology of hidradenitis suppurativa; in Jemec GBE, Revuz J, Leyden JJ (ed): Hidradenitis Suppurativa. Berlin, Springer, 2006.
Combination Therapy with Clindamycin and Rifampicin for HS
Dermatology 2009;219:148-154 
12 Sartorius K, Lapins J, Emtestam L, Jemec GB: Suggestions for uniform outcome variables when reporting treatment effects in hidradenitis suppurativa. Br J Dermatol 2003;149: 211-213.

13 Revuz J: Modifications et mode d'emploi du score de Sartorius pour évaluer la gravité de l'hidradénite suppurée. Ann Dermatol Venereol 2007;134:173-174.

14 Hurley HJ: Axillary hyperhidrosis, apocrine bromhidrosis, hidradenitis suppurativa, and familial benign pemphigus: surgical approach; in Roenigk RK, Roenigk HH, (eds) Dermatologic Surgery. New York, Dekker 1989, pp 729-739.

-15 Van der Zee HH, Boer J, Prens EP, Jemec GBE: The effect of combined treatment with oral clindamycin and oral rifampicin in patients with hidradenitis suppurativa. Dermatology 2009;219:143-147.

16 Canoui-Poitrine F, Revuz J, Wolkenstein P, Viallette C, Gabison G, Pouget F, Poli F, Faye O, Bastuji-Garin S: Clinical characteristics of a series of 302 French patients suffering from hidradenitis suppurativa, with an analysis of factors associated with disease severity. J Am Acad Dermatol 2009, in press.

17 Von der Werth JM, Jemec GBE: Morbidity in patients with hidradenitis suppurativa. Br J Dermatol 2001;144:809-813.

18 Jemec GBE, Heidenheim M, Nielsen NH: Hidradenitis suppurativa - characteristics and consequences. Clin Exp Dermatol 1996;21: 419-423.

19 Tsankov N, Angelova I: Rifampicin in dermatology. Clin Dermatol 2003;21:50-55.

20 Arditi M, Yogev R: In vitro interaction between rifampin and clindamycin against pathogenic coagulase-negative staphylococci. Antimicrob Agents Chemother 1989;33: 245-247.
21 Spizek J, Rezanka T: Lincomycin, clindamycin and their applications. Appl Microbiol Biotechnol 2004;64:455-464.

22 Clemmensen OJ: Topical treatment of hidradenitis suppurativa with clindamycin. Int J Dermatol 1983;22:325-328.

23 Jemec GB, Wendelboe P: Topical clindamycin versus systemic tetracycline in the treatment of hidradenitis suppurativa. J Am Acad Dermatol 1998;39:971-974.

24 Schlievert PM, Kelly JA: Clindamycin-induced suppression of toxic-shock syndromeassociated exotoxin production. J Infect Dis 1984;149:471.

25 Wijaya A, Wulansari R, Ano H, Makimura S: Effect of clindamycin therapy on phagocytic and oxidative activity profiles of spleen mononuclear cells in Babesia rodhaini-infected mice. J Vet Med Sci 2001;63:563-566.

-26 Spisani S, Traniello S, Martuccio C, Rizzuti O, Cellai L: Rifamycins inhibit human neutrophil functions: new derivatives with potential antiinflammatory activity. Inflammation 1997;21:391-400.

27 Liu XG, Narang PK, Li RC: Induction of hepatic and presystemic metabolism of antipyrine in the mice: rifampicin versus rifabutin Eur J Drug Metab Pharmacokinet 2001;26: 209-213.

28 Yerramasetti R, Gollapudi S, Gupta S: Rifampicin inhibits CD95-mediated apoptosis of Jurkat $\mathrm{T}$ cells via glucocorticoid receptors by modifying the expression of molecules regulating apoptosis. J Clin Immunol 2002; 22:37-47.
29 Sullivan TP, Welsh E, Kerdel FA, Burdick AE, Kirsner RS: Infliximab for hidradenitis suppurativa. Br J Dermatol 2003;149:10461049.

30 Moschella SL: Is there a role for infliximab in the current therapy of hidradenitis suppurativa? A report of three treated cases. Int J Dermatol 2007;46:1287-1291.

31 Mekkes JR, Bos JD: Long-term efficacy of a single course of infliximab in hidradenitis suppurativa. Br J Dermatol 2008;158:370374.

32 Fernández-Vozmediano JM, Armario-Hita JC: Infliximab for the treatment of hidradenitis suppurativa. Dermatology 2007;215: 41-44.

-33 Fardet L, Dupuy A, Kerob D, Levy A, Allez M, Begon E, Bachelez H, Morel P, Lebbé C: Infliximab for severe hidradenitis suppurativa: transient clinical efficacy in 7 consecutive patients. J Am Acad Dermatol 2007;56: 624-628.

34 Thielen AM, Barde C, Saurat JH: Long-term infliximab for severe hidradenitis suppurativa. Br J Dermatol 2006;155:1105-1107.

35 Cusack C, Buckley C: Etanercept: effective in the management of hidradenitis suppurativa. Br J Dermatol 2006;154:726-729.

36 Giamarellos-Bourboulis EJ, Pelekanou E, Antonopoulou A, Petropoulou H, Baziaka F, Karagianni V, Stavrianeas N, Giamarellou $\mathrm{H}$ : An open-label phase II study of the safety and efficacy of etanercept for the therapy of hidradenitis suppurativa. $\mathrm{Br} \mathrm{J}$ Dermatol 2008;158:567-572.

-37 Scheinfeld N: Treatment of coincident seronegative arthritis and hidradenitis suppurativa with adalimumab. J Am Acad Dermatol 2006;55:163-164.

38 Moul DK, Korman NJ: The cutting edge: severe hidradenitis suppurativa treated with adalimumab. Arch Dermatol 2006;142: 1110-1112. 